\title{
Statistical study of medium-scale traveling ionospheric disturbances using SuperDARN Hokkaido ground backscatter data for 2011
}

\author{
Alexey V Oinats ${ }^{1 *}$, Vladimir I Kurkin ${ }^{1}$ and Nozomu Nishitani ${ }^{2}$
}

\begin{abstract}
We describe an automated technique to determine parameters of traveling ionospheric disturbances (TIDs) using the Super Dual Auroral Radar Network (SuperDARN) high frequency (HF) radar data. The technique is based on the analysis of minimum ground backscatter range variations corresponding to different radar beams. Using this technique, we processed the SuperDARN Hokkaido radar data for 2011 and revealed statistical distributions of medium-scale TID (MSTID) azimuth and apparent horizontal velocity. We found four peaks with a distinct diurnal and seasonal dependence in the MSTID azimuth occurrence rate distributions. Northeast MSTID azimuths $\left(20^{\circ}\right.$ to $\left.50^{\circ}\right)$ are typical of the summer and equinox morning hours; southeast azimuths $\left(100^{\circ}\right.$ to $\left.140^{\circ}\right)$ prevail in the winter daytime; southwest azimuths $\left(190^{\circ}\right.$ to $220^{\circ}$ ) are typical mostly in the summer and equinox nighttime and in the equinox evening; northwest azimuths $\left(280^{\circ}\right.$ to $\left.320^{\circ}\right)$ are typical of the summer daytime and evening. The apparent horizontal velocities are generally within the 100 to $160 \mathrm{~m} / \mathrm{s}$ range. The obtained results agree well with earlier studies by other researchers. However, there are also certain differences. The summer daytime northwestward MSTIDs are not indicated in the earlier studies. The nighttime horizontal velocities are 1.5 to 2 times higher than those in the daytime. Furthermore, winter velocity values are about 1.5 times higher than those in other seasons. These differences might be associated with the peculiarities of the data recorded by different facilities, or the features of the processing techniques, and require further investigation for their interpretation.
\end{abstract}

Keywords: Medium-scale traveling ionospheric disturbances; Atmospheric gravity waves; SuperDARN Hokkaido radar

\section{Correspondence/findings Introduction}

By traveling ionospheric disturbances (TIDs), we understand wave-like electron density disturbances caused by atmospheric gravity waves (AGWs) propagating at ionospheric heights. One should distinguish between medium-scale and large-scale TIDs (Hunsucker 1982). Medium-scale TIDs (MSTIDs) have horizontal wavelengths of about several hundreds of kilometers and periods of $15 \mathrm{~min}$ up to $1 \mathrm{~h}$. Large-scale TIDs (LSTIDs) have horizontal wavelengths of more than $1,000 \mathrm{~km}$ and periods of $30 \mathrm{~min}$ to $3 \mathrm{~h}$.

TIDs have been studied for decades (Hocke and Schlegel 1996; Hunsucker 1982), including studies that have used

\footnotetext{
* Correspondence: oinats@iszf.irk.ru

${ }^{1}$ Institute of Solar-Terrestrial Physics, Siberian Branch of the Russian Academy of Sciences, 664033, Lermontova St., 126a, PO Box 291, Irkutsk, Russia Full list of author information is available at the end of the article
}

the Super Dual Auroral Radar Network (SuperDARN) high frequency (HF) radar data (Bristow and Greenwald 1995; Bristow et al. 1994; Chisham et al. 2007; Karhunen et al. 2006; Samson et al. 1990; Ogawa et al. 2012). A TID passage within the radar field-of-view leads to HF wave focusing/defocusing effects. The latter manifests themselves in periodic variations of the received ground backscatter power (Samson et al. 1990). One of the ground backscatter characteristics most sensitive to TIDs and measured by SuperDARN radar is the minimum range corresponding to the skip distance (Arnold et al. 1998; Stocker et al. 2000). Karhunen et al. (2006) proposed a technique involving a special multi-frequency mode (METSO). This technique uses skip distance measurements to build a two-dimensional phase map of the disturbance. From this map, one can obtain the azimuth, horizontal velocity, and horizontal wavelength as a function of the disturbance period. 
In contrast to different case studies examining the properties of individual TIDs, statistical studies, using wide datasets, enable us to reveal the general pattern of AGW propagation. A number of papers presenting statistical studies of TIDs have been published in the last 15 years (Afraimovich et al. 1999, 2000; Ding et al. 2008; Kotake et al. 2007; Medvedev et al. 2013; Otsuka et al. 2011; Shiokawa et al. 2003; Tsugawa et al. 2004). There are also statistical studies that utilized SuperDARN radar data (Bristow et al. 1996; He et al. 2004; Ichihara et al. 2013; Ishida et al. 2008; Grocott et al. 2013). Apparently, a relatively small number of such studies are related to the complexity of extensive dataset processing, as well as the issues concerning an automated interpretation of HF radar data.

In this paper, we present a statistical analysis of MSTID azimuth and apparent horizontal velocity calculated using the SuperDARN Hokkaido HF radar data obtained in 2011. We used an automated technique where a cross-correlation analysis of minimum ground backscatter range variations is used to determine TID parameters. We did not place limitations on the spatial scale or temporal periodicity of the observed disturbances, but we analyzed the minimum range variations as they were observed. A statistical sampling for the crosscorrelation function calculation is varied within the 1.5 to $7 \mathrm{~h}$ interval. Therefore, both MSTIDs and LSTIDs are included in the analysis, but we suppose the LSTID effect is negligible in our statistical results because LSTIDs are observed about an order of magnitude less frequently. In addition, we focused on investigating the seasonal and diurnal dependence of the above parameters to compare them with other recent statistical studies.

\section{Instrumentation and analysis method}

We used the 2011 SuperDARN Hokkaido HF radar $\left(43.53^{\circ} \mathrm{N}, 143.61^{\circ} \mathrm{E}\right)$ ground backscatter data in this study (http://center.stelab.nagoya-u.ac.jp/hokkaido/index.html). The phased-array antenna system enables the radiation pattern to be focused in one of 16 azimuth directions (narrow beam with approximately $3.5^{\circ}$ width). The radar measures echoes from ranges of up to approximately $3,500 \mathrm{~km}$. Figure 1 shows a map of the radar location, as well as all 16 beam directions that form the approximately 50-degree-wide field-of-view. In the standard operational mode, the radar continuously scans the entire field-ofview. Scanning takes either 1 or $2 \mathrm{~min}$, then the process is repeated depending upon the integration time along each beam, which is 3 or $6 \mathrm{~s}$, respectively. The operating frequency is selected from the allowed frequency range and remains relatively constant throughout a day. We analyzed the data obtained at the approximately $11 \mathrm{MHz}$ operating frequency because it was mostly used in 2011. The SuperDARN Hokkaido radar

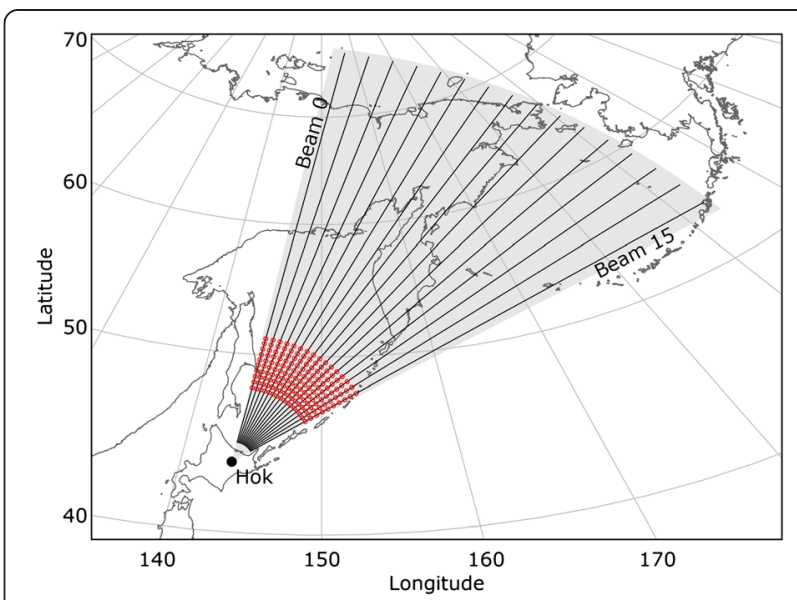

Figure 1 Map of SuperDARN Hokkaido radar location and its field-of-view. The radar beam directions and field-of-view are shown by diverging black lines and gray sector, respectively. Approximate locations of ionospheric reflection points are shown by red circles.

measures range-time dependencies of the following echo parameters at each beam: power, Doppler velocity, Doppler spectral width, and elevation angle.

Ground backscatter is one of two echo types recorded by the radar. In addition to ground backscatter echoes, there are also echoes backscattered directly by ionospheric irregularities. Separation between the two echo types is performed in the standard way (He et al. 2004). The ground backscatter echoes are supposed to have a Doppler velocity and Doppler spectral width of typically less than 50 and $30 \mathrm{~m} / \mathrm{s}$, respectively. Figure 2a illustrates an example of the SuperDARN Hokkaido data, showing the ground backscatter echo power received by the radar along Beam 0 versus slant range and Japanese standard time (JST $=\mathrm{UT}+9 \mathrm{~h}$ ) on November 24, 2011. The oblique lines with enhanced power clearly seen in Figure 2a manifest the MSTID passage through the radar field-of-view.

\section{Data preprocessing}

As shown by Milan et al. (1997), SuperDARN radars can receive ground backscatter echoes not only from the front but also from behind the radar. In the case of the Hokkaido radar, echoes from behind might be observed more frequently during daytime at low solar activity, when the critical frequency is reduced so that there is no ionospheric reflection in front of the radar. However, HF waves may be reflected from behind the radar from northern slope of the equatorial anomaly, where the critical frequency is greater. To exclude the echoes coming from the rear field-of-view, additional interferometer data (elevation angle) processing is provided, according to Milan et al. (1997). 


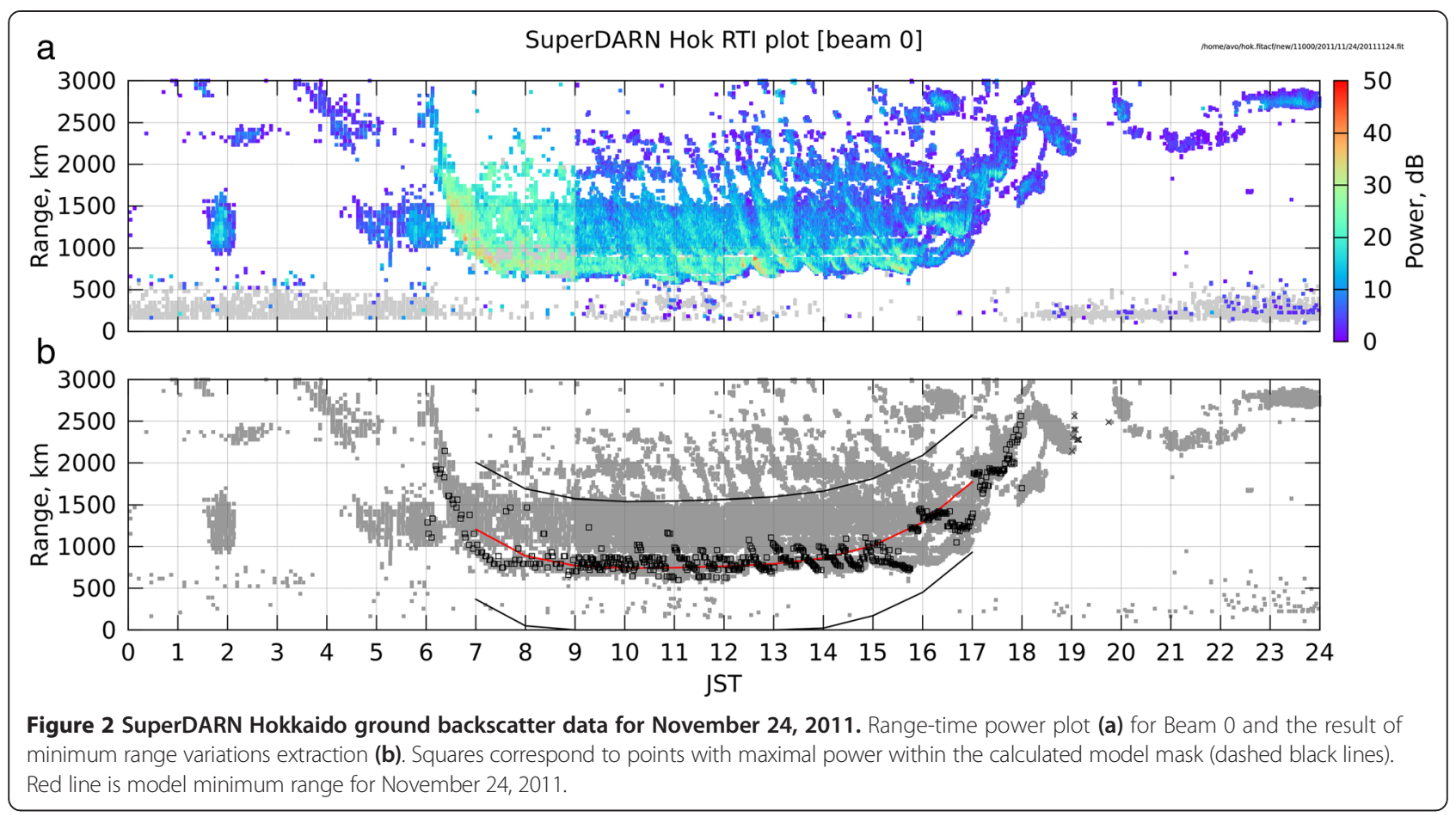

We are interested in MSTIDs propagating at the heights of the F2 ionospheric layer. Except for the regular one-hop propagation ground backscatter echoes reflected by the F2 ionospheric layer, one may also record other echoes. Among them, there are regular echoes reflected by the E layer, as well as two-hop propagation echoes reflected by the E and F2 layers. In Figure $2 \mathrm{a}$, a regular F2 one-hop propagation echo is seen at 6:00 through 18:00 JST within the 700 to $1,700 \mathrm{~km}$ range, simultaneously with an F2 two-hop propagation echo (above approximately $1,800 \mathrm{~km}$ range), and with other (irregular) echoes at nighttime. To correctly exclude the echoes not related to the F2 one-hop propagation, we developed a model of ground backscatter characteristics. It describes diurnal and seasonal variations of the F2 one-hop propagation ground backscatter characteristics for all beams and for the main operating frequencies of the SuperDARN Hokkaido radar. The model is based on the ground backscatter simulation using the original software developed within the waveguide approach and the International Reference Ionosphere model (Bilitza and Reinisch 2008). The simulation was performed for the 15th day of each month. Figure 3 shows, for example, diurnal and seasonal dependencies of four model parameters: minimum range, skip distance, elevation angle, and ratio between the total range to ground scatter and the range to the ionospheric reflection point (the ray trajectory apogee). Figure 3 is a plot for Beam 0, with an operating frequency of $11 \mathrm{MHz}$, for December 2010 through January 2012. The range ratio (see Figure $3 \mathrm{~d}$ ) characterizes the asymmetry of the ray trajectory caused by the regular ionospheric gradients. As we can see for poleward trajectories, this ratio is greater than two. In addition, the model does not predict ground backscatter echoes at nighttime in October to April. This is because of the low nighttime critical frequency during these months, resulting in the HF wave passing through the ionosphere without reflection. By linear interpolation, the model allows us to calculate a mask in which the regular ground backscatter echo should be for each day of the year.

Further preprocessing is illustrated in Figure 2b, where the red and black lines show the calculated model minimum range and mask, respectively, for Beam 0 on November 24, 2011. The squares show the ranges where the maxima of the received ground backscatter power were found within the mask at each moment in time. The squares represent the required dependence of the slant range versus time. We fit the dependencies for all beams by penalized regression splines (not shown in Figure 2b) which is found to be good because of adaptive control over smoothing (Ruppert et al. 2003). Then, using splines, we recalculate the dependencies on an equidistant time grid common for all beams. As a final step, we remove the diurnal trend from the dependencies to obtain the minimum range variations corresponding to the TID effects.

\section{Technique for TID parameter estimation}

The technique for TID parameter estimation (Oinats et al. 2012, 2013) is based on three assumptions. The first two assumptions, concerning the TID's model, are 

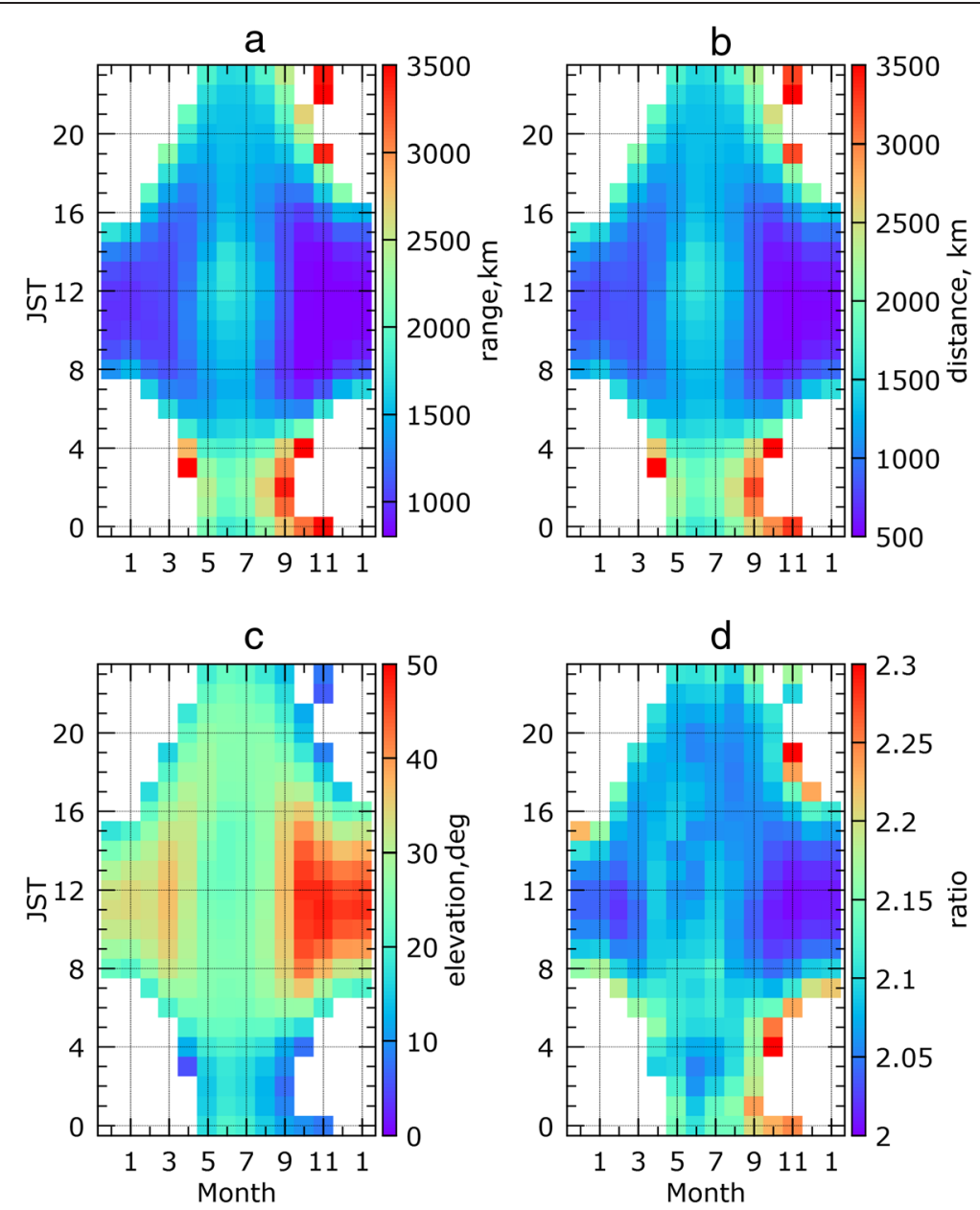

Figure 3 Diurnal and seasonal model for ground backscatter characteristics. For Beam 0 and operational frequency of $11 \mathrm{MHz}$ in 2011. Minimum range (a), corresponding skip distance $(\mathbf{b})$, elevation angle (c), and range ratio (d), characterizing asymmetry of the trajectory.

that a disturbance has a plane phase front within the radar field-of-view, and the phase velocity vertical component is equal to zero. The third assumption is that the ionosphere mainly influences the ground backscatter at the apogee of the propagation trajectory, i.e., where reflection occurs. Using the third assumption, we can consider the minimum range variations caused by ionosphere variations that occur directly in the reflection point. Figure 1 shows the approximate location of the ionospheric reflection points for the SuperDARN Hokkaido radar (red circles).

The disturbance's phase is a function of observational point radius vector, $\vec{r}$, and of time, $t$. According to the first assumption, we have:

$$
\phi(\vec{r}, t)=\vec{k} \vec{r}-\omega t+\phi_{0}
$$

where $\vec{k}$ and $\omega$ are the TID wave vector and cyclic frequency, respectively, and $\phi_{0}$ is an initial phase. The radar field-of-view is about $50^{\circ}$ in the azimuth direction. This corresponds to about $500 \mathrm{~km}$ between reflection points for Beams 0 and 15. Therefore, we should write Expression 1 in terms of spherical coordinates with regard to the radar location. For the case of zero vertical phase velocity, we can write:

$$
\phi(\theta, \varphi, \mathrm{t})=k r_{0}\left[\left(\varphi-\varphi^{\prime}\right) \cos \theta \sin \Phi+\left(\theta-\theta^{\prime}\right) \cos \Phi\right]-\omega t+\phi_{0}
$$

where $\Phi$ is the TID azimuth (clockwise from north), $k=|\vec{k}|=2 \pi / \lambda, \lambda$ is the TID spatial wavelength, $\left(\theta^{\prime}, \varphi^{\prime}\right)$ are the latitude and longitude of the radar location, and $r_{0}$ is the Earth's radius. Thus, the time lag between variations corresponding to the $i$ th and $j$ th radar beams is given by expression (Oinats et al. 2013):

$$
\begin{gathered}
\Delta t_{j i}(\Phi, v)=\frac{r_{0}}{v}\left[\left(\left(\varphi_{j}-\varphi^{\prime}\right) \cos \theta_{j}-\left(\varphi_{i}-\varphi^{\prime}\right) \cos \theta_{i}\right) \sin \Phi\right. \\
\left.+\left(\theta_{j}-\theta_{i}\right) \cos \Phi\right]
\end{gathered}
$$


where $v=\omega / \mathrm{k}$ is the TID phase velocity, and $\left(\theta_{\mathrm{i}}, \varphi_{\mathrm{i}}\right)$ and $\left(\theta_{\mathrm{j}}, \varphi_{\mathrm{i}}\right)$ are the coordinates (latitude and longitude) of the reflection points for the $i$ th and $j$ th beams. Excluding the $i=j$ case, we can obtain 240 equations of type Equation 3 with two unknown parameters for all 16 radar beams. Certainly, one needs only two combined equations to determine two unknown parameters. This corresponds to measuring the variations at three reflection points. However, we should keep in mind the possible inaccuracy of data processing. For example, an ambiguity might arise when minimum range variations are extracted from the radar data. The coordinates of the reflection points are also determined approximately. Therefore, the solution for the equation set is found through two-dimensional least-squares fitting. The discrepancy between the observed and the theoretical time lags is given by:

$$
\delta(\Phi, v)=\sum_{j, i=0, j \neq i}^{15}\left(\Delta t_{j i}^{\prime}-\Delta \mathrm{t}_{j i}(\Phi, v)\right)^{2}
$$

The observed time lag, $\Delta t_{j i}^{\prime}$, for each pair of variations is determined as a time shift corresponding to the maximum of the cross-correlation function. The statistical sampling size corresponds to the prevailing period in the variation's spectrum within the 0.8 to $5 \mathrm{~h}$ interval.

To determine the coordinates of the reflection points, we first calculate the ground distance to the reflection point using the slant range and the well-known formulas based on Breit and Tuve's theorem (Davies 1990). Two parameters are needed: the slant range to the ionospheric reflection point and either the effective reflection height or the elevation angle. Unfortunately, the elevation angle measurements made by the Hokkaido radar have not as yet been calibrated. Therefore, we assume the effective height of the ionospheric reflection is constant at $250 \mathrm{~km}$ for all ranges (we found that this value was appropriate for the 2011 Hokkaido radar data). Such an approach is quite reasonable and has been used earlier by other researchers (Bristow et al. 1994; Grocott et al. 2013). In addition, as shown by Karhunen et al. (2006), even when sounding is done at different operating frequencies, the ionospheric reflection height remains approximately the same.

The slant range to the reflection point is equal to half of the slant range to the ground scatter in the case of a spherically symmetric ionosphere. However, in general, there might be an asymmetry of the trajectory due to ionospheric gradients. Our estimations show that the difference for the ground distance to the reflection point can reach up to $15 \%$ because of the regular ionospheric gradients for the 2011 Hokkaido radar data. This leads to the emergence of corresponding errors in the TID horizontal velocity estimations, especially for diurnal intervals, when the solar terminator passes through the radar field-of-view. To minimize the error, we calculate the slant range to the reflection point using the model range ratio (see Figure 3d). Finally, we calculate the appropriate latitude and longitude with reference to the beam azimuth and the radar coordinates.

In Equation 4, we take into account only those time lags, for which the correlation coefficient exceeds the 0.85 threshold. Minimization of the discrepancy is performed using the Levenberg-Marquardt algorithm for solving nonlinear least squares problems. If the number of the time lags satisfying the mentioned threshold is less than three, minimization is not performed.

The above processing was carried out for each local time. In Figure $4 \mathrm{a}$, the calculated diurnal variations of the azimuth and horizontal velocity are shown for November 24, 2011 (see Figure 2). As one can see, the azimuth and horizontal velocity vary during the day. There are 'jumps' that can be connected with uncertainties in time lag calculations because of the presence of components with smaller periods in the variations. However, the common diurnal trends are seen clearly enough. The azimuth in the morning hours is relatively stable, at about $210^{\circ}$, then changes rapidly to approximately $120^{\circ}$ at 10:00 JST, then increases again to approximately $220^{\circ}$. The disturbance propagation direction changes clockwise from southeast to southwest during the daytime. The velocity increases from approximately $150 \mathrm{~m} / \mathrm{s}$ in the morning to approximately $350 \mathrm{~m} / \mathrm{s}$ in the evening. Figure $4 \mathrm{~b}, \mathrm{c}$ shows the corresponding distributions of the azimuths and velocities, respectively. Because the parameters vary during the day, there are no single maxima in the distributions, but the ranges of parameter change are clear enough.

\section{Results and discussion}

All the SuperDARN Hokkaido radar data for 2011 were processed in the manner described above. Figure 5 shows the relative occurrence rate of azimuths and of apparent horizontal velocities for the entire year. Each bar in the distribution is proportional to the number of cases for which the calculated azimuth (or velocity) is within the given 10-degree-width interval (or $20 \mathrm{~m} / \mathrm{s}$ ). Only the variations for which the average root mean square (RMS) over all beams exceeded $60 \mathrm{~km}$ were taken into account (thus, the variation amplitude exceeds the radar range gate). As seen in Figure $5 \mathrm{a}$, there are four dominant MSTID propagation directions: northeast (approximately $35^{\circ}$ ), southeast (approximately $120^{\circ}$ ), southwest (approximately $205^{\circ}$ ), and northwest (approximately $295^{\circ}$ ). The relative occurrence rate for these directions is approximately 1.5 to 2 times higher than the background rate. The most frequent horizontal velocities are within the 100 to $160 \mathrm{~m} / \mathrm{s}$ range. 

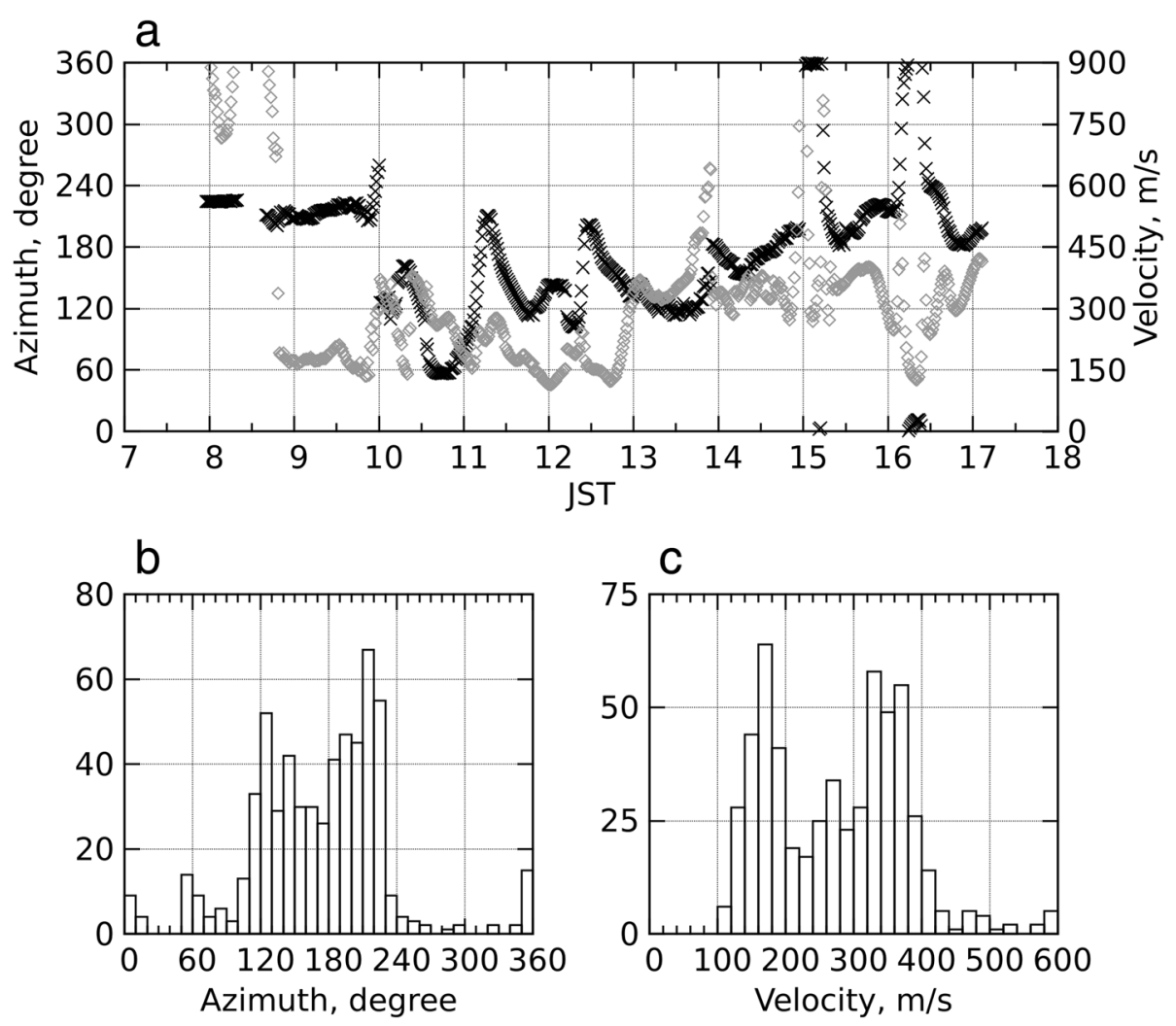

Figure 4 Result of TID azimuth and horizontal velocity calculation for the data presented in Figure 2. Time dependence (a) of azimuth (black crosses) and velocity (gray diamonds) and azimuth (b) and velocity (c) distributions.

To study the diurnal dependence, we calculated the relative occurrence rate over each hour of the diurnal interval as was done by Kotake et al. (2007) and Otsuka et al. (2011), who used the GPS total electron content (TEC) maps over Japan (GEONET) to determine MSTID parameters. Figures $6 \mathrm{a}$ and $7 \mathrm{a}$ show the results for the whole year for azimuth and velocity, respectively.
As we can see, there is a distinct diurnal dependence of the four dominant MSTID groups. Azimuths corresponding to the northeast peak $\left(20^{\circ}\right.$ to $\left.50^{\circ}\right)$ are typical of the morning hours (4 to 6 JST). Southeast azimuths $\left(100^{\circ}\right.$ to $\left.140^{\circ}\right)$ prevail in the daytime (8 to $13 \mathrm{JST}$ ). Southwest azimuths $\left(190^{\circ}\right.$ to $\left.220^{\circ}\right)$ are typical mostly at night and in the evening (16 to 2 JST). Northwest
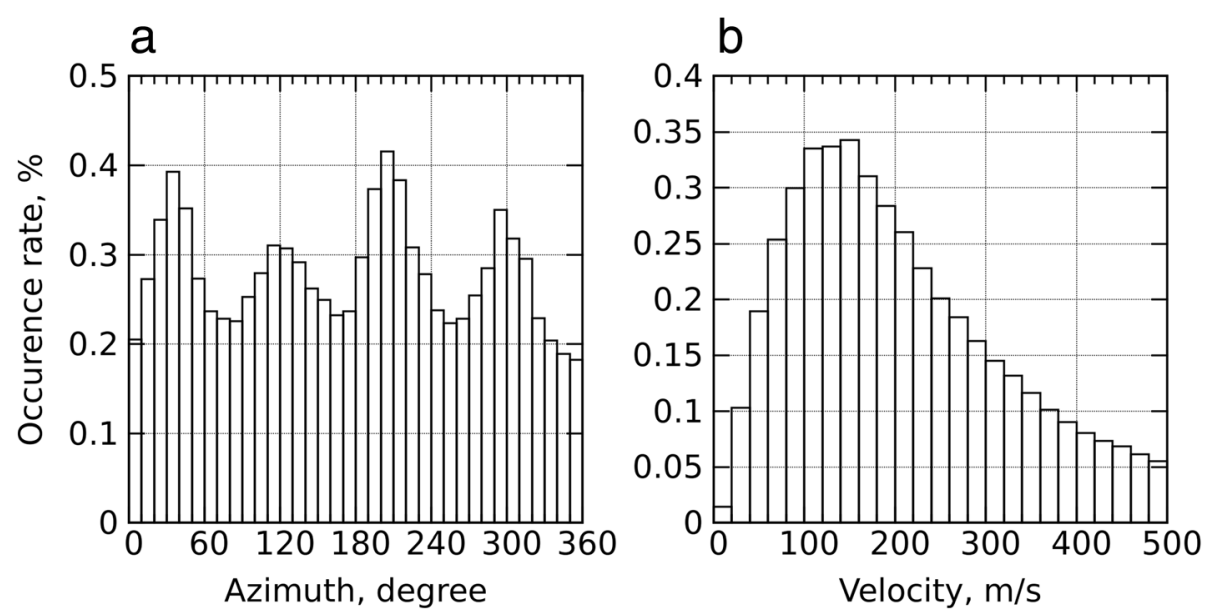

Figure 5 Relative occurrence rate of calculated azimuths (a) and of apparent horizontal velocities (b) in 2011. 


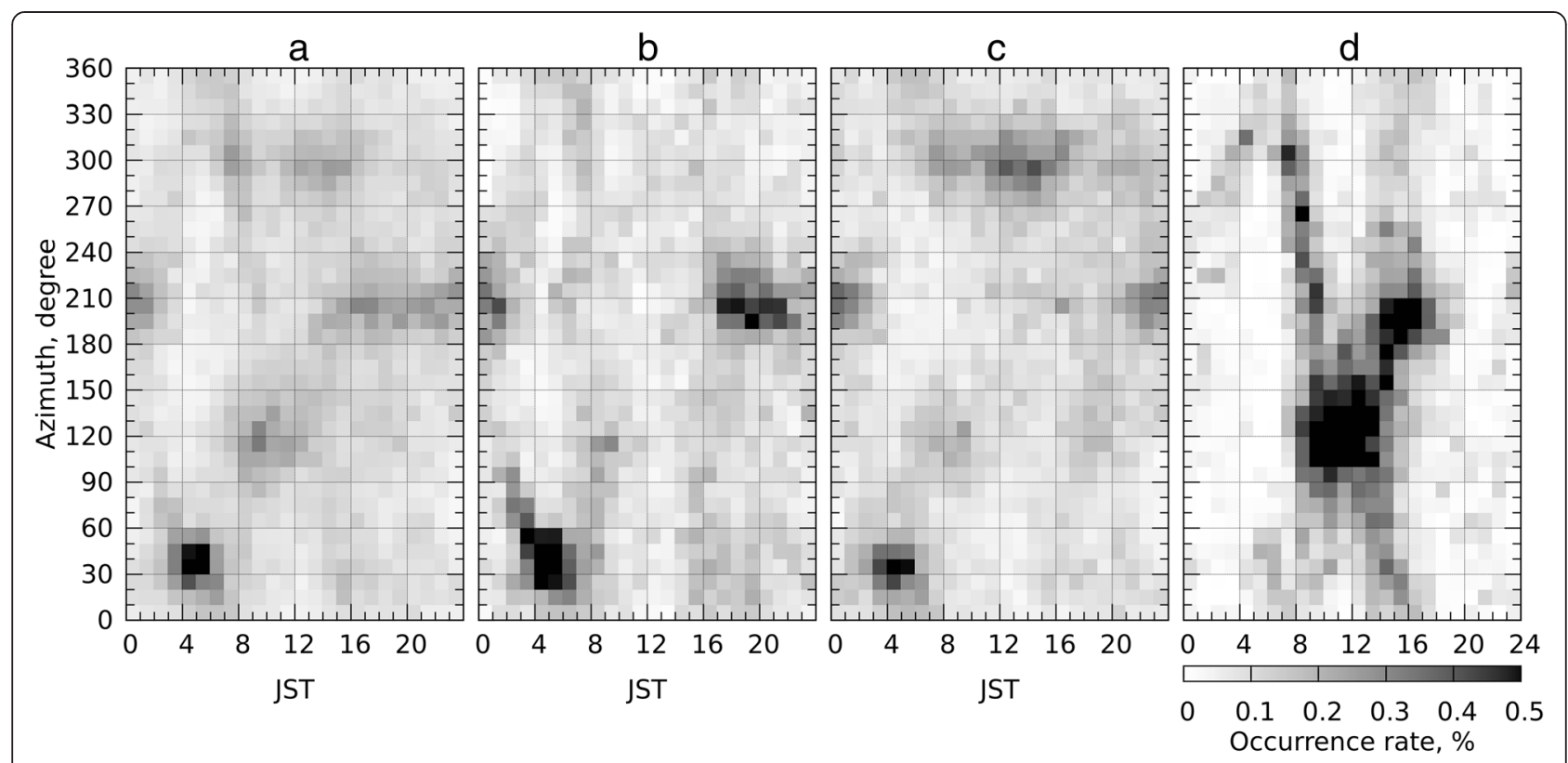

Figure 6 Local time relative occurrence rate of calculated TID azimuths in 2011. For entire year (a), equinoxes (b), summer (c), and winter (d).

azimuths $\left(280^{\circ}\right.$ to $\left.320^{\circ}\right)$ are typical of daytime and in the evening (12 to 17 JST).

We calculated similar distributions separately for three seasons in 2011: equinox (March, April, September, and October), summer (May, June, July, and August) and winter (November, December, January, and February). As seen in Figure 6d, the daytime southward MSTIDs are most typical of the winter. Furthermore, the propagation direction of such disturbances changes clockwise during the day from the southeast (approximately $100^{\circ}$ ) at 9 JST to the southwest (approximately $210^{\circ}$ ) at 17 JST. Such behavior of daytime MSTIDs was indicated earlier and is well explained by the filtering properties of the neutral wind (Afraimovich et al. 1999; Kotake et al. 2007). However, Otsuka et al. (2011) did not find such a distinct dependence for the Japan region, indicating only the south-southeast direction for the winter daytime MSTIDs. Ishida et al. (2008) also indicated the dominant south-southeast direction for the winter daytime MSTIDs.

As seen in Figure 6b,c, the nighttime southwestward MSTIDs are typical of the summer and equinoxes. Other authors (Afraimovich et al. 1999; Kotake et al. 2007; Otsuka et al. 2011) found that such MSTIDs were also typical in winter. Ichihara et al. (2013) noted that approximately two thirds of the nighttime MSTIDs

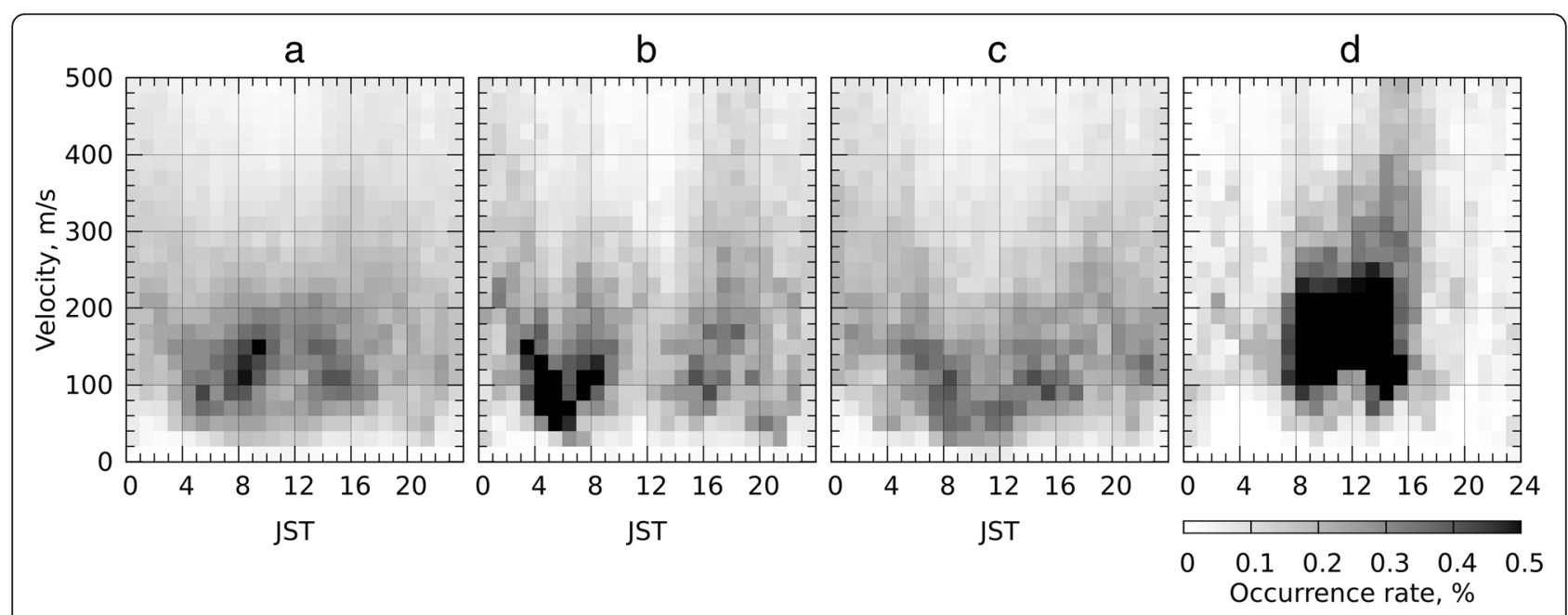

Figure 7 Local time relative occurrence rate of calculated TID apparent horizontal velocity in 2011. For entire year (a), equinoxes (b), summer (c), and winter (d). 
propagate southwestward, regardless of the season. Southwestward propagation of most of the observed nighttime MSTIDs was indicated by Shiokawa et al. (2003), who used the 630-nm airglow images at two stations in Japan from 1998 till 2000. The nighttime southwestward MSTIDs were also mentioned by Medvedev et al. (2013), who analyzed the Irkutsk incoherent scatter radar and the ionosonde DPS-4 data using the technique for full TID wave vector determination. The occurrence rate is seen to be weak in the winter nighttime (see Figure 6d) because the regular ground backscatter echoes are almost absent during this period. Morning northeastward MSTIDs are observed mainly in the summer and equinoxes (see Figure 6b,c, respectively). The evening northwestward MSTIDs are typical of summer (see Figure 6c). This is also consistent with Kotake et al. (2007) and Otsuka et al. (2011).

Thus, our results agree well with previous studies. However, there are also several differences. According to Figure $6 \mathrm{c}$, the northwestward MSTIDs are typical of the summer daytime. Other studies do not indicate this. As clearly seen in Figure 7a, the horizontal velocity nighttime values are 1.5 to 2 times higher than those for the daytime. According to Medvedev et al. (2013), the absolute velocity nighttime values are approximately two times higher than those for the daytime. However, there is no diurnal dependence found for the apparent horizontal velocity. It is also interesting that, according to Figure $7 \mathrm{~d}$, the horizontal velocities in winter are about 1.5 times greater than those in other seasons. Explanation for the mentioned differences requires further investigation. Most likely, these differences can be related to the peculiarities of the data recorded by different facilities or the features of the processing techniques. In particular, one of the issues with the Hokkaido radar is an absence of calibrated elevation angle measurements. This leads to difficulties in accurate identification of the overlapping E and F2 ground backscatter echoes, which is the case during the summer daytime. Another related issue is the rough mapping of the ionospheric reflection points. The latter mainly affects the accuracy of the horizontal velocity determination as discussed by Grocott et al. (2013).

\section{Conclusion}

We developed an original automated technique for SuperDARN HF radar data processing to determine the main TID parameters. The technique is based on crosscorrelation analysis of the ground backscatter minimum range variations at different radar beams. The 2011 SuperDARN Hokkaido radar dataset was processed using this technique. We calculated the occurrence rates of the MSTID azimuth and of MSTID apparent horizontal velocity for the entire year, as well as for different seasons, depending on the local time.

There are four peaks with a distinct diurnal and seasonal dependence in the MSTID azimuth occurrence rate distributions. Northeast MSTID azimuths $\left(20^{\circ}\right.$ to $\left.50^{\circ}\right)$ are typical of the summer and equinox morning hours; southeast azimuths $\left(100^{\circ}\right.$ to $\left.140^{\circ}\right)$ prevail in the winter daytimes; southwest azimuths $\left(190^{\circ}\right.$ to $\left.220^{\circ}\right)$ are typical mostly in the summer, and equinox nighttime and in the equinox evening; northwest azimuths $\left(280^{\circ}\right.$ to $320^{\circ}$ ) are typical of the summer daytime and evening. The apparent horizontal velocities are generally within the $100^{\circ}$ to $160 \mathrm{~m} / \mathrm{s}$ range. The results agree well with earlier studies by other researchers. However, there are several differences whose explanation requires further investigation.

The technique described here can be applied to the extensive SuperDARN Hokkaido radar dataset, as well as to the data of other SuperDARN radars. Such a study, if performed, could be useful for better understanding of AGW propagation and generation mechanisms.

\section{Abbreviations}

AGW: atmospheric gravity waves; HF: high frequency; LSTID: large-scale traveling ionospheric disturbance; MSTID: medium-scale traveling ionospheric disturbance; SuperDARN: Super Dual Auroral Radar Network; TID: traveling ionospheric disturbance.

\section{Competing interests}

The authors declare that they have no competing interests.

\section{Authors' contributions}

$\mathrm{AO}$ elaborated the technique of TID parameter estimation, provided the processing and calculations, and drafted the manuscript. VK proposed the idea of the study and supervised the work of AO at ISTP. NN provided the Hokkaido SuperDARN radar data, supervised the work of AO at STEL, and participated in the drafting the manuscript. All the authors participated in the discussion and interpretation of the obtained statistical results. All authors read and approved the final manuscript.

\section{Acknowledgements}

We would like to thank Dr. K. G. Ratovsky and Dr. A. V. Medvedev at the Institute of Solar-Terrestrial Physics of the Siberian Branch of the Russian Academy of Sciences for useful discussions and helpful comments. We are very grateful to the referees for their appropriate and constructive suggestions. This study was done with financial support from the Russian Foundation for Basic Research (under grants No. 14-05-00259, No. 14-05-00588, and No. 12-05-00865-a).

\section{Author details}

${ }^{1}$ Institute of Solar-Terrestrial Physics, Siberian Branch of the Russian Academy of Sciences, 664033, Lermontova St., 126a, PO Box 291, Irkutsk, Russia.

${ }^{2}$ Solar-Terrestrial Environment Laboratory, Nagoya University, Furo-cho, Chikusa-ku, Nagoya 464-8601, Japan.

Received: 30 April 2014 Accepted: 23 January 2015

Published online: 15 February 2015

\section{References}

Afraimovich EL, Boitman ON, Zhovty El, Kalikhman AD, Pirog TG (1999) Dynamics and anisotropy of traveling ionospheric disturbances as deduced from transionospheric sounding data. Radio Sci 34:477-487

Afraimovich EL, Lipko W, Vugmeister B (2000) Determining dynamic parameters of different-scale ionospheric irregularities over northern Siberia. J Atmos Sol-Terr Phys 62:133-140 
Arnold NF, Jones T, Robinson T (1998) Validation of the CUTLASS HF radar gravity wave observing capability using EISCAT CP-1 data. Ann Geophys 16:1392-1399

Bilitza D, Reinisch BW (2008) International reference ionosphere 2007: improvements and new parameters. Adv Space Res 42:599-609, doi:10.1016/j.asr.2007.07.048

Bristow WA, Greenwald RA (1995) Estimating gravity wave parameters from oblique high-frequency backscatter: modeling and analysis. J Geophys Res 100(A3):3639-3648

Bristow WA, Greenwald RA, Samson JC (1994) Identification of high-latitude acoustic gravity wave sources using the Goose Bay HF radar. J Geophys Res 99(A1):319-331

Bristow WA, Greenwald RA, Villain JP (1996) On the seasonal dependence of medium-scale atmospheric gravity waves in the upper atmosphere at high latitudes. J Geophys Res 101(A7):15685-15699

Chisham G, Lester M, Milan SE, Freeman MP, Bristow WA, Grocott A, McWilliams KA, Ruohoniemi JM, Yeoman TK, Dyson PL, Greenwald RA, Kikuchi T, Pinnock M, Rash JPS, Sato N, Sofko GJ, Villain J-P, Walker ADM (2007) A decade of the Super Dual Auroral Radar Network (SuperDARN): scientific achievements, new techniques and future directions. Surv Geophys 28:33-109, doi:10.1007/s10712-007-9017-8

Davies K (1990) lonospheric radio. Peter Peregrinus Ltd, London

Ding F, Wan W, Liu L, Afraimovich EL, Voeykov SV, Perevalova NP (2008) A statistical study of large-scale traveling ionospheric disturbances observed by GPS TEC during major magnetic storms over the years 2003-2005. J Geophys Res. doi:10.1029/2008JA013037

Grocott A, Hosokawa K, Ishida T, Lester M, Milan SE, Freeman MP, Sato N, Yukimatu AS (2013) Characteristics of medium-scale traveling ionospheric disturbances observed near the Antarctic Peninsula by HF radar. J Geophys Res Space Phys 118:5830-5841, doi:10.1002/jgra.50515

He L-S, Dyson P, Parkinson ML, Wan W (2004) Studies of medium scale travelling ionospheric disturbances using TIGER SuperDARN radar sea echo observations. Ann Geophys 22:4077-4088

Hocke K, Schlegel K (1996) A review of atmospheric gravity waves and travelling ionospheric disturbances: 1982-1995. Ann Geophys 14:917-940

Hunsucker RD (1982) Atmospheric gravity waves generated in the high-latitude ionosphere: a review. Rev Geophys Space Phys 20(2):293-315, doi:10.1029/RG020i002p00293

Ichihara A, Nishitani N, Ogawa T, Tsugawa T (2013) Northward-propagating nighttime medium-scale traveling ionospheric disturbances observed with SuperDARN Hokkaido HF radar and GEONET. Adv Polar Sci 24:42-49, doi:10.3724/SP.J.1085.2013.00042

Ishida T, Hosokawa K, Shibata T, Suzuki S, Nishitani N, Ogawa T (2008) SuperDARN observations of daytime MSTIDs in the auroral and mid-latitudes: possibility of longdistance propagation. Geophys Res Lett 35:L13102, doi:10.1029/2008GL034623

Karhunen TJT, Robinson TR, Arnold NF, Lester M (2006) Determination of the parameters of travelling ionospheric disturbances in the high-latitude ionosphere using CUTLASS coherent scatter radars. J Atmos Sol-Terr Phys 68:558-567, doi:10.1016/j.jastp.2005.03.021

Kotake N, Otsuka Y, Ogawa T, Tsugawa T, Saito A (2007) Statistical study of medium-scale traveling ionospheric disturbances observed with the GPS networks in Southern California. Earth Planets Space 59:95-102

Medvedev AV, Ratovsky KG, Tolstikov MV, Alsatkin SS, Scherbakov AA (2013) Studying of the spatial-temporal structure of wavelike ionospheric disturbances on the base of Irkutsk incoherent scatter radar and Digisonde data. J Atmos Sol-Terr Phys 105-106:350-357, doi:10.1016/j.jastp.2013.09.001

Milan SE, Jones TB, Robinson TR, Thomas EC, Yeoman TK (1997) Interferometric evidence for the observation of ground backscatter originating behind the CUTLASS coherent HF radars. Ann Geophys 15:29-39

Ogawa T, Nishitani N, Tsugawa T, Shiokawa K (2012) Giant ionospheric disturbances observed with the SuperDARN Hokkaido HF radar and GPS network after the 2011 Tohoku earthquake. Earth Planets Space 64(12):1295-1307

Oinats AV, Kurkin VI, Kutelev KA, Nishitani N (2012) The outlook of SuperDARN radars application for monitoring of the ionospheric dynamics in Russia. Physical Bases of Instrumentation 1(3):3-18 (in Russian)

Oinats AV, Kurkin VI, Nishitani N, Saito A (2013) On the determination of traveling ionospheric disturbances parameters using SuperDARN radar data. Electromagnetic Waves and Electronic Systems 18(8):30-39 (in Russian)

Otsuka Y, Kotake N, Shiokawa K, Ogawa T, Tsugawa T, Saito A (2011) Statistical study of medium-scale traveling ionospheric disturbances observed with a GPS receiver network in Japan. In: Abdu M, Pancheva D, Bhattacharyya A (eds) Aeronomy of the Earth's atmosphere and ionosphere. Springer Netherlands, Dordrecht
Ruppert D, Wand MP, Carroll RJ (2003) Semiparametric regression. Cambridge University Press, New York

Samson JC, Greenwald RAA, Ruohoniemi JM, Frey A, Baker KB (1990) Goose Bay radar observations of Earth-reflected, atmospheric gravity waves in the high-latitude ionosphere. J Geophys Res 95(A6):7693-7709

Shiokawa K, Ihara C, Otsuka Y, Ogawa T (2003) Statistical study of nighttime medium-scale traveling ionospheric disturbances using midlatitude airglow images. J Geophys Res 108(A1):1052, doi:10.1029/2002JA009491

Stocker AJ, Arnold NF, Jones TB (2000) The synthesis of travelling ionospheric disturbance (TID) signatures in HF radar observations using ray tracing. Ann Geophys 18:56-64, doi:10.1007/s00585-000-0056-4

Tsugawa T, Saito A, Otsuka Y (2004) A statistical study of large-scale traveling ionospheric disturbances using the GPS network in Japan. J Geophys Res 109:A06302, doi:10.1029/2003JA010302

\section{Submit your manuscript to a SpringerOpen ${ }^{\odot}$ journal and benefit from:}

- Convenient online submission

- Rigorous peer review

- Immediate publication on acceptance

- Open access: articles freely available online

- High visibility within the field

- Retaining the copyright to your article

Submit your next manuscript at $>$ springeropen.com 